\title{
Remark on the Off-Diagonal Expansion of the Bergman Kernel on Compact Kähler Manifolds
}

\author{
Xiaonan Ma • George Marinescu
}

Received: 25 February 2013 / Accepted: 26 February 2013 / Published online: 15 March 2013 (C) School of Mathematical Sciences, University of Science and Technology of China and Springer-Verlag Berlin Heidelberg 2013

\begin{abstract}
In this short note, we compare our previous work on the off-diagonal expansion of the Bergman kernel and the preprint of Lu-Shiffman (arXiv:1301.2166). In particular, we note that the vanishing of the coefficient of $p^{-1 / 2}$ is implicitly contained in Dai-Liu-Ma's work (J. Differ. Geom. 72(1), 1-41, 2006) and was explicitly stated in our book (Holomorphic Morse inequalities and Bergman kernels. Progress in Math., vol. 254, 2007).
\end{abstract}

Keywords Kähler manifold · Bergman kernel of a positive line bundle

Mathematics Subject Classification (2010) 53C55 - 53C21 · 53D50 - 58J60

In this short note we revisit the calculations of some coefficients of the off-diagonal expansion of the Bergman kernel from our previous work [4, 5].

Let $(X, \omega)$ be a compact Kähler manifold of $\operatorname{dim}_{\mathbb{C}} X=n$ with Kähler form $\omega$. Let $\left(L, h^{L}\right)$ be a holomorphic Hermitian line bundle on $X$, and let $\left(E, h^{E}\right)$ be a holomorphic Hermitian vector bundle on $X$. Let $\nabla^{L}, \nabla^{E}$ be the holomorphic Hermitian connections on $\left(L, h^{L}\right),\left(E, h^{E}\right)$ with curvatures $R^{L}=\left(\nabla^{L}\right)^{2}, R^{E}=\left(\nabla^{E}\right)^{2}$, respectively. We assume that $\left(L, h^{L}, \nabla^{L}\right)$ is a prequantum line bundle, i.e., $\omega=\frac{\sqrt{-1}}{2 \pi} R^{L}$.

X. Ma $(\bowtie)$

UFR de Mathématiques, Université Paris Diderot - Paris 7, Case 7012, 75205 Paris Cedex 13, France

e-mail:ma@math.jussieu.fr

G. Marinescu

Mathematisches Institut, Universität zu Köln, Weyertal 86-90, 50931 Köln, Germany

e-mail: gmarines@math.uni-koeln.de

G. Marinescu

Institute of Mathematics 'Simion Stoilow', Romanian Academy, Bucharest, Romania 
Let $P_{p}\left(x, x^{\prime}\right)$ be the Bergman kernel of $L^{p} \otimes E$ with respect to $h^{L}, h^{E}$ and the Riemannian volume form $d v_{X}=\omega^{n} / n$ !. This is the integral kernel of the orthogonal projection from $\mathscr{C}^{\infty}\left(X, L^{p} \otimes E\right)$ to the space of holomorphic sections $H^{0}\left(X, L^{p} \otimes E\right)$ (cf. [4, §4.1.1]).

We fix $x_{0} \in X$. We identify the ball $B^{T_{x_{0}} X}(0, \varepsilon)$ in the tangent space $T_{x_{0}} X$ to the ball $B^{X}\left(x_{0}, \varepsilon\right)$ in $X$ by the exponential map (cf. [4, §4.1.3]). For $Z \in B^{T_{x_{0}} X}(0, \varepsilon)$ we identify $\left(L_{Z}, h_{Z}^{L}\right),\left(E_{Z}, h_{Z}^{E}\right)$ to $\left(L_{x_{0}}, h_{x_{0}}^{L}\right),\left(E_{x_{0}}, h_{x_{0}}^{E}\right)$ by parallel transport with respect to the connections $\nabla^{L}, \nabla^{E}$ along the curve $\gamma_{Z}:[0,1] \ni u \rightarrow \exp _{x_{0}}^{X}(u Z)$. Then $P_{p}\left(x, x^{\prime}\right)$ induces a smooth section $\left(Z, Z^{\prime}\right) \mapsto P_{p, x_{0}}\left(Z, Z^{\prime}\right)$ of $\pi^{*} \operatorname{End}(E)$ over $\left\{\left(Z, Z^{\prime}\right) \in T X \times_{X} T X:|Z|,\left|Z^{\prime}\right|<\varepsilon\right\}$, which depends smoothly on $x_{0}$, with $\pi: T X \times_{X} T X \rightarrow X$ the natural projection. If $d v_{T X}$ is the Riemannian volume form on $\left(T_{x_{0}} X, g^{T_{x_{0}}} X\right)$, there exists a smooth positive function $\kappa_{x_{0}}: T_{x_{0}} X \rightarrow \mathbb{R}$, defined by

$$
d v_{X}(Z)=\kappa_{x_{0}}(Z) d v_{T X}(Z), \quad \kappa_{x_{0}}(0)=1 .
$$

For $Z \in T_{x_{0}} X \cong \mathbb{R}^{2 n}$, we denote $z_{j}=Z_{2 j-1}+\sqrt{-1} Z_{2 j-1}$ its complex coordinates, and set

$$
\mathscr{P}\left(Z, Z^{\prime}\right)=\exp \left(-\frac{\pi}{2} \sum_{i=1}^{n}\left(\left|z_{i}\right|^{2}+\left|z_{i}^{\prime}\right|^{2}-2 z_{i} \bar{z}_{i}^{\prime}\right)\right) .
$$

The near off-diagonal asymptotic expansion of the Bergman kernel in the form established [4, Theorem 4.1.24] is the following.

Theorem 1 Given $k, m^{\prime} \in \mathbb{N}, \sigma>0$, there exists $C>0$ such that if $p \geqslant 1, x_{0} \in X$, $Z, Z^{\prime} \in T_{x_{0}} X,|Z|,\left|Z^{\prime}\right| \leqslant \sigma / \sqrt{p}$,

$$
\left|\frac{1}{p^{n}} P_{p}\left(Z, Z^{\prime}\right)-\sum_{r=0}^{k} \mathscr{F} r\left(\sqrt{p} Z, \sqrt{p} Z^{\prime}\right) \kappa^{-\frac{1}{2}}(Z) \kappa^{-\frac{1}{2}}\left(Z^{\prime}\right) p^{-\frac{r}{2}}\right|_{\mathscr{C}^{m^{\prime}}(X)} \leqslant C p^{-\frac{k+1}{2}} .
$$

where $\mathscr{C}^{m^{\prime}}(X)$ is the $\mathscr{C}^{m^{\prime}}$-norm with respect to the parameter $x_{0}$,

$$
\mathscr{F}_{r}\left(Z, Z^{\prime}\right)=J_{r}\left(Z, Z^{\prime}\right) \mathscr{P}\left(Z, Z^{\prime}\right)
$$

$J_{r}\left(Z, Z^{\prime}\right) \in \operatorname{End}(E)_{x_{0}}$ are polynomials in $Z, Z^{\prime}$ with the same parity as $r$ and $\operatorname{deg} J_{r}\left(Z, Z^{\prime}\right) \leqslant 3 r$, whose coefficients are polynomials in $R^{T X}$ (the curvature of the Levi-Civita connection on $T X), R^{E}$ and their derivatives of order $\leqslant r-2$.

Remark 2 For the above properties of $J_{r}\left(Z, Z^{\prime}\right)$ see [4, Theorem 4.1.21 and end of $\S 4.1 .8]$. They are also given in [2, Theorem 4.6, (4.107) and (4.117)]. Moreover, by [4, (1.2.19) and (4.1.28)], $\kappa$ has a Taylor expansion with coefficients the derivatives of $R^{T X}$. As in [4, (4.1.101)] or [5, Lemma 3.1 and (3.27)] we have

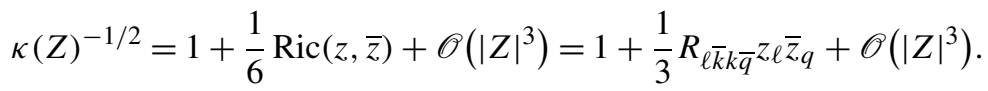

Note that a more powerful result than the near-off diagonal expansion from Theorem 1 holds. Namely, by [2, Theorem 4.18'] and [4, Theorem 4.2.1], the full offdiagonal expansion of the Bergman kernel holds (even for symplectic manifolds), 
i.e., an analogous result to (3) for $|Z|,\left|Z^{\prime}\right| \leqslant \varepsilon$. This appears naturally in the proof of the diagonal expansion of the Bergman kernel on orbifolds in [2, (5.25)] or [4, (5.4.14), (5.4.23)].

Proposition 3 The coefficient $\mathscr{F}_{1}$ vanishes identically: $\mathscr{F}_{1}\left(Z, Z^{\prime}\right)=0$ for all $Z, Z^{\prime}$. Therefore the coefficient of $p^{-1 / 2}$ in the expansion of $p^{-n} P_{p}\left(p^{-1 / 2} Z, p^{-1 / 2} Z^{\prime}\right)$ vanishes, so the latter converges to $\mathscr{F}_{0}\left(Z, Z^{\prime}\right)$ at rate $p^{-1}$ as $p \rightarrow \infty$.

Proof This is [4, Remark 4.1.26] or [5, (2.19)], see also [2, (4.107), (4.117), (5.4)].

When $E=\mathbb{C}$ with trivial metric, the vanishing of $\mathscr{F}_{1}$ was recently rediscovered in [3, Theorem 2.1] $\left(b_{1}(u, v)=0\right.$ therein). In [3] an equivalent formulation [6] of the expansion (3) is used, based on the analysis of the Szegö kernel from [1]. In [3, Theorem 2.1] further off-diagonal coefficients $\mathscr{F}_{2}, \mathscr{F}_{3}, \mathscr{F}_{4}$ are calculated in the $K$-coordinates. From [5, (3.22)], we see that the usual normal coordinates are $K$ coordinates up to order at least 3 . This shows that the vanishing of $\mathcal{F}_{1}$ given by Proposition 3 implies the vanishing of $b_{1}$ calculated with the help of in $K$-coordinates. We wish to point out that we calculated in [5] the coefficients $\mathscr{F}_{1}, \ldots, \mathscr{F}_{4}$ on the diagonal, using the off-diagonal expansion (3) and evaluating $\mathscr{F}_{r}$ for $Z=Z^{\prime}=0$. Thus, off-diagonal formulas for $\mathscr{F}_{1}, \ldots, \mathscr{F}_{4}$ are implicitly contained in [5]. We show below how the coefficient $\mathscr{F}_{2}$ can be calculated in the framework of [5].

We use the notation in [5, (3.6)], then $r=8 R_{m \bar{q} q \bar{m}}$ is the scalar curvature.

Proposition 4 The coefficient $J_{2}$ in (4) is given by

$$
\begin{aligned}
J_{2}\left(Z, Z^{\prime}\right)= & -\frac{\pi}{12} R_{k \bar{m} \ell \bar{q}}\left(z_{k} z_{\ell} \bar{z}_{m} \bar{z}_{q}+6 z_{k} z_{\ell} \bar{z}_{m}^{\prime} \bar{z}_{q}^{\prime}-4 z_{k} z_{\ell} \bar{z}_{m} \bar{z}_{q}^{\prime}\right. \\
& \left.-4 z_{k} z_{\ell}^{\prime} \bar{z}_{m}^{\prime} \bar{z}_{q}^{\prime}+z_{k}^{\prime} z_{\ell}^{\prime} \bar{z}_{m}^{\prime} \bar{z}_{q}^{\prime}\right) \\
& -\frac{1}{3} R_{k \bar{m} q \bar{q}}\left(z_{k} \bar{z}_{m}+z_{k}^{\prime} \bar{z}_{m}^{\prime}\right)+\frac{1}{8 \pi} \boldsymbol{r}+\frac{1}{\pi} R_{q \bar{q}}^{E} \\
& -\frac{1}{2}\left(z_{\ell} \bar{z}_{q}-2 z_{\ell} \bar{z}_{q}^{\prime}+z_{\ell}^{\prime} \bar{z}_{q}^{\prime}\right) R_{\ell \bar{q}}^{E} .
\end{aligned}
$$

Remark 5 Setting $Z=Z^{\prime}=0$ in (6) we obtain the coefficient $\boldsymbol{b}_{1}\left(x_{0}\right)=J_{2}(0,0)=$ $\frac{1}{8 \pi} \boldsymbol{r}+\frac{1}{\pi} R_{q \bar{q}}^{E}$ of $p^{-1}$ of the (diagonal) expansion of $p^{-n} P_{p}\left(x_{0}, x_{0}\right)$, cf. [4, Theorem 4.1.2].

Moreover, in order to obtain the coefficient of $p^{-1}$ in the expansion (3) we multiply $\mathscr{F}_{2}\left(\sqrt{p} Z, \sqrt{p} Z^{\prime}\right)$ to the expansion of $\kappa(Z)^{-1 / 2} \kappa\left(Z^{\prime}\right)^{-1 / 2}$ with respect to the variable $\sqrt{p} Z$ obtained from (5). If $E=\mathbb{C}$ the result is a polynomial which is the sum of a homogeneous polynomial of order four and a constant, similar to [3]. 
Proof of Proposition 4 Set

$$
\begin{aligned}
& b_{i}=-2 \frac{\partial}{\partial z_{i}}+\pi \bar{z}_{i}, \quad b_{i}^{+}=2 \frac{\partial}{\partial \bar{z}_{i}}+\pi z_{i}, \quad \mathscr{L}=\sum_{i=1}^{n} b_{i} b_{i}^{+}, \\
& \widetilde{\mathcal{O}_{2}}=\frac{b_{m} b_{q}}{48 \pi} R_{k \bar{m} \ell \bar{q} z_{k} z_{l}}+\frac{b_{q}}{3 \pi} R_{\ell \bar{k} k \bar{q}} z_{\ell}-\frac{b_{q}}{12} R_{k \bar{m} \ell \bar{q} z_{k} z_{\ell} \bar{z}_{m}^{\prime} .} .
\end{aligned}
$$

By $[4,(4.1 .107)]$ or $[5,(2.19)]$, we have

$$
\mathscr{F}_{2, x_{0}}=-\mathscr{L}^{-1} \mathscr{P}^{\perp} \mathcal{O}_{2} \mathscr{P}-\mathscr{P} \mathcal{O}_{2} \mathscr{L}^{-1} \mathscr{P}^{\perp} .
$$

By [5, (4.1a), (4.7)] we have

$$
\left(\mathscr{L}^{-1} \mathscr{P}^{\perp} \mathcal{O}_{2} \mathscr{P}\right)\left(Z, Z^{\prime}\right)=\left(\mathscr{L}^{-1} \mathcal{O}_{2} \mathscr{P}\right)\left(Z, Z^{\prime}\right)=\left\{\widetilde{\mathcal{O}_{2}}+\frac{b_{q}}{4 \pi} R_{\ell \bar{q}}^{E} z_{\ell}\right\} \mathscr{P}\left(Z, Z^{\prime}\right) .
$$

By the symmetry properties of the curvature [5, Lemma 3.1] we have

$$
R_{k \bar{m} \ell \bar{q}}=R_{\ell \bar{m} k \bar{q}}=R_{k \bar{q} \ell \bar{m}}=R_{\ell \bar{q} k \bar{m}}, \quad \overline{R_{k \bar{m} \ell \bar{q}}}=R_{m \bar{k} q \bar{\ell}}, \quad\left(R_{k \bar{q}}^{E}\right)^{*}=R_{q \bar{k}}^{E} .
$$

We use throughout that $\left[g(z, \bar{z}), b_{j}\right]=2 \frac{\partial}{\partial z_{j}} g(z, \bar{z})$ for any polynomial $g(z, \bar{z})$ (cf. $[5$, (1.7)]). Hence from (10), we get

$$
\begin{aligned}
& b_{q} R_{k \bar{k} \ell \bar{q}} z_{\ell}=R_{k \bar{k} \ell \bar{q}} z_{\ell} b_{q}-2 R_{k \bar{k} q \bar{q}}, \\
& b_{q} R_{k \bar{m}} \ell \bar{q} z_{k} z_{\ell}=-4 R_{k \bar{m}} \bar{q} z_{k}+R_{k \bar{m}} \ell \bar{q} z_{k} z_{\ell} b_{q},
\end{aligned}
$$

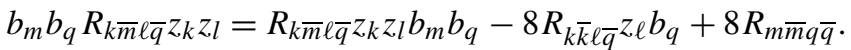

Thus from (7) and (11), we get

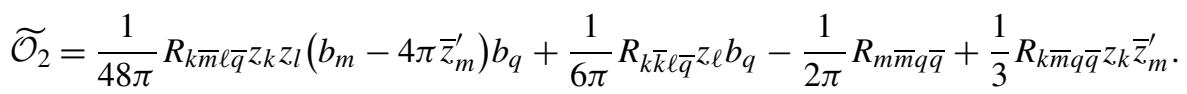

Now, $\left(b_{i} \mathscr{P}\right)\left(Z, Z^{\prime}\right)=2 \pi\left(\bar{z}_{i}-\bar{z}_{i}^{\prime}\right) \mathscr{P}\left(Z, Z^{\prime}\right)$, see [4, (4.1.108)] or [5, (4.2)]. Therefore

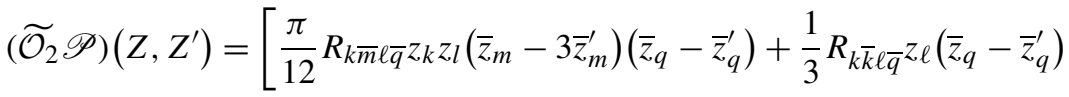

$$
\begin{aligned}
& \left.-\frac{1}{2 \pi} R_{m \bar{m} q \bar{q}}+\frac{1}{3} R_{k \bar{m}} q \bar{q} z_{k} \bar{z}_{m}^{\prime}\right] \mathscr{P}\left(Z, Z^{\prime}\right)
\end{aligned}
$$

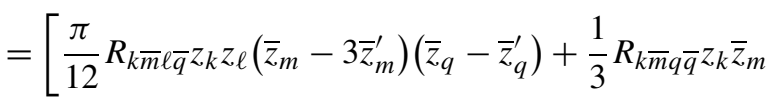

$$
\begin{aligned}
& \left.-\frac{1}{2 \pi} R_{m \bar{m} q \bar{q}}\right] \mathscr{P}\left(Z, Z^{\prime}\right) \text {. }
\end{aligned}
$$


We know that for an operator $T$ we have $T^{*}\left(Z, Z^{\prime}\right)=\overline{T\left(Z^{\prime}, Z\right)}$, thus

$$
\begin{aligned}
\left(\widetilde{\mathcal{O}_{2}} \mathscr{P}\right)^{*}\left(Z, Z^{\prime}\right)= & {\left[\frac{\pi}{12} R_{k \bar{m} \ell} \bar{z}_{m}^{\prime} \bar{z}_{q}^{\prime}\left(z_{k}^{\prime}-3 z_{k}\right)\left(z_{\ell}^{\prime}-z_{\ell}\right)+\frac{1}{3} R_{k \bar{m}} q \bar{q} \bar{z}_{m}^{\prime} z_{k}^{\prime}\right.} \\
& \left.-\frac{1}{2 \pi} R_{m \bar{m} q \bar{q}}\right] \mathscr{P}\left(Z, Z^{\prime}\right) .
\end{aligned}
$$

We have $\left(\mathscr{P} \mathcal{O}_{2} \mathscr{L}^{-1} \mathscr{P} \perp\right)^{*}=\mathscr{L}^{-1} \mathscr{P} \perp \mathcal{O}_{2} \mathscr{P}$ by [4, Theorem 4.1.8], so from (13) and (14), we obtain the factor of $R_{k \bar{m} \ell \bar{q}}$ in (6).

Let us calculate the contribution of the last term (curvature of $E$ ). We have

$$
-\left(\frac{b_{q}}{4 \pi} R_{\ell \bar{q}}^{E} z_{\ell} \mathscr{P}\right)\left(Z, Z^{\prime}\right)=\left(\frac{1}{2 \pi} R_{q \bar{q}}^{E}-\frac{1}{2} z_{\ell}\left(\bar{z}_{q}-\bar{z}_{q}^{\prime}\right) R_{\ell \bar{q}}^{E}\right) \mathscr{P}\left(Z, Z^{\prime}\right)
$$

and by (10), we also have

$$
-\left(\frac{b_{q}}{4 \pi} R_{\ell \bar{q} z_{\ell}}^{E} \mathscr{P}\right)^{*}\left(Z, Z^{\prime}\right)=\left(\frac{1}{2 \pi} R_{q \bar{q}}^{E}-\frac{1}{2} \bar{z}_{\ell}^{\prime}\left(z_{q}^{\prime}-z_{q}\right) R_{q \bar{\ell}}^{E}\right) \mathscr{P}\left(Z, Z^{\prime}\right) .
$$

The contribution to $J_{2}$ of the term on $E$ is thus given by the last two terms in (6).

Acknowledgements X. Ma partially supported by Institut Universitaire de France. G. Marinescu partially supported by DFG funded projects SFB/TR 12 and MA 2469/2-2.

\section{References}

1. Boutet de Monvel, L., Sjöstrand, J.: Sur la singularité des noyaux de Bergman et de Szegö. In: Journées Équations aux Dérivées Partielles de Rennes (1975). Astérisque, vol. 34-35, pp. 123-164. Soc. Math. France, Paris (1976)

2. Dai, X., Liu, K., Ma, X.: On the asymptotic expansion of Bergman kernel. J. Differ. Geom. 72(1), 1-41 (2006). Announced in C. R. Math. Acad. Sci. Paris 339(3), 193-198 (2004)

3. Lu, Z., Shiffman, B.: Asymptotic expansion of the off-diagonal Bergman kernel on compact Kähler manifolds. Preprint arXiv:1301.2166

4. Ma, X., Marinescu, G.: Holomorphic Morse Inequalities and Bergman Kernels. Progress in Math., vol. 254, Birkhäuser, Basel (2007), xiii, 422 p.

5. Ma, X., Marinescu, G.: Berezin-Toeplitz quantization on Kähler manifolds. J. Reine Angew. Math. 662, 1-56 (2012)

6. Shiffman, B., Zelditch, S.: Asymptotics of almost holomorphic sections of ample line bundles on symplectic manifolds. J. Reine Angew. Math. 544, 181-222 (2002) 